\title{
Investigating clinical heterogeneity in systematic reviews: a methodologic review of guidance in the literature
}

\author{
Joel J Gagnier ${ }^{1 *}$, David Moher ${ }^{2,3}$, Heather Boon ${ }^{4}$, Joseph Beyene ${ }^{5}$ and Claire Bombardier ${ }^{6}$
}

\begin{abstract}
Background: While there is some consensus on methods for investigating statistical and methodological heterogeneity, little attention has been paid to clinical aspects of heterogeneity. The objective of this study is to summarize and collate suggested methods for investigating clinical heterogeneity in systematic reviews.
\end{abstract}

Methods: We searched databases (Medline, EMBASE, CINAHL, Cochrane Library, and CONSORT, to December 2010) and reference lists and contacted experts to identify resources providing suggestions for investigating clinical heterogeneity between controlled clinical trials included in systematic reviews. We extracted recommendations, assessed resources for risk of bias, and collated the recommendations.

Results: One hundred and one resources were collected, including narrative reviews, methodological reviews, statistical methods papers, and textbooks. These resources generally had a low risk of bias, but there was minimal consensus among them. Resources suggested that planned investigations of clinical heterogeneity should be made explicit in the protocol of the review; clinical experts should be included on the review team; a set of clinical covariates should be chosen considering variables from the participant level, intervention level, outcome level, research setting, or others unique to the research question; covariates should have a clear scientific rationale; there should be a sufficient number of trials per covariate; and results of any such investigations should be interpreted with caution.

Conclusions: Though the consensus was minimal, there were many recommendations in the literature for investigating clinical heterogeneity in systematic reviews. Formal recommendations for investigating clinical heterogeneity in systematic reviews of controlled trials are required.

\section{Background}

Systematic reviews sometimes apply statistical techniques to combine data from multiple studies resulting in a meta-analysis. Meta-analyses result in a point estimate, the summary treatment effect, together with a measure of the precision of results (e.g., a 95\% confidence interval). These measures of precision represent the degree of variability or heterogeneity in the results among included studies. There are several possible sources of variability or heterogeneity among studies that are included in meta-analyses. Variability in the participants, the types or timing of outcome measurements, and

\footnotetext{
* Correspondence: jgagnier@umich.edu

${ }^{1}$ Departments of Orthopaedic Surgery and Epidemiology, University of

Michigan, 24 Frank Lloyd Wright Drive, Ann Arbor, MI, USA

Full list of author information is available at the end of the article
}

intervention characteristics may be termed clinical heterogeneity; variability in the trial design and quality is typically termed methodological heterogeneity; variability in summary treatment effects between trials is termed statistical heterogeneity [1]. Methodological and clinical sources of heterogeneity contribute to the magnitude and presence of statistical heterogeneity [1].

Methodological heterogeneity hinges on aspects of implementation of the individual trials and how they differ from each other. For example, trials that do not adequately conceal allocation to treatment groups may result in overestimates in the meta-analytic treatment effects [2]. Significant statistical heterogeneity arising from methodological heterogeneity suggests that the studies are not all estimating the same effects due to different degrees of bias. 
Clinical heterogeneity arises from differences in participant characteristics (e.g., sex, age, baseline disease severity, ethnicity, comorbidities), types or timing of outcome measurements, and intervention characteristics (e.g., dose and frequency of dose [1]). This heterogeneity can cause significant statistical heterogeneity, inaccurate summary effects and associated conclusions, misleading decision makers and others. As such, systematic reviewers need to consider how best to handle sources of heterogeneity [1]. For example, preplanned subgroup analyses, stratifying for similar characteristics of the intervention and participants, could tease-out important scientific and clinically relevant information [3].

Systematic reviews are frequently recognized as the best available evidence for decisions about health-care management and policy [3-7]. Results of systematic reviews are often incorporated into clinical practice guidelines [5] and required in funding applications by granting agencies [6]. In spite of all this it appears health-care professionals and policy makers infrequently use systematic reviews to guide decision-making [8].

A limitation of many systematic reviews is that their content and format are frequently not useful to decision makers [8]. For example, while some guidance exists describing what to include in reports of systematic reviews (e.g., the PRISMA statement [9]), characteristics of the intervention that are necessary to apply their findings are infrequently provided [10-13]. This has led to some preliminary work on how to extract clinically relevant information from systematic reviews [14]. Furthermore, systematic reviews commonly show substantial heterogeneity in estimated effects (statistical heterogeneity), possibly due to methodological, clinical or unknown features in the included trials [15]. While guidance exists on the assessment and investigation of methodological [1] and statistical heterogeneity $[1,16]$, little attention has been given to clinical heterogeneity.

We report a systematic review of suggested methods for investigating clinical heterogeneity in systematic reviews of controlled clinical trials. We also provide some guidance for systematic reviewers.

\section{Methods}

This project identified resources giving recommendations for investigating clinical heterogeneity in systematic reviews. We extracted their recommendations, assessed their risk for bias, and categorized and described the suggestions.

\section{Search}

The following databases were searched: Medline (to October 29, 2010), EMBASE (to Oct 30, 2010), CINAHL (1981 to Oct 30, 2010), Health Technology Assessment (to Oct 29, 2010), the Cochrane Methodology Register (to Oct 29, 2010), and the CONSORT database of methodological papers (to October 30, 2010). A library and information scientist was consulted to create sensitive and specific searches, combining appropriate terms and extracting new terms from relevant studies for each database. The following search terms were used in the various databases and at various stages of the search: heterogeneity, applicability, clinical, assessment, checklist, guideline(s), scale, and criteria. The "adjacent" or "within X words" tools were used for the terms "clinical" and "heterogeneity" for all databases. In addition, we used the PubMed related-links option that identifies indexed studies on similar topics or having similar indexing terms to include a broad range of papers that might be indirectly related to clinical heterogeneity. Appendix A contains details of the electronic searching. One investigator (JG) contacted representatives of the Cochrane Collaboration, the Campbell Collaboration, the Agency for Healthcare Research and Quality (AHRQ) and a selection of experts identified through an initial review of the literature to suggest relevant articles, guidelines, position papers, textbooks or other experts in the area. We also reviewed the Cochrane Handbook, the Campbell Collaboration methods guides, and the AHRQ comparative effectiveness section for any guidance on clinical heterogeneity and searched reference lists of all retrieved resources.

The overall process consisted of a "snowballing" technique of seeking information on the topic, by which we asked experts to refer us to other experts or resources, and so on, until each new resource yielded a negligible return. Several individuals with expertise in the area of systematic reviews (JG, DM, JB, CB) met to identify key textbooks to include out of thier personal knowledge of textbooks in the area. These individuals presented what each felt were key textbooks in the area and then debated the merits of each, finally coming to a consensus-based decision on which to include. In general, these methods allowed us to include a broad array of resources related to investigating clinical heterogeneity in systematic reviews.

\section{Inclusion criteria}

Clinical heterogeneity is defined as differences in participant, treatment, or outcome characteristics or research setting. We included any methodological study, systematic review, guideline, textbook, handbook, checklist, scale, or other published guidance document with a focus on assessing, measuring, or generally investigating clinical heterogeneity between or within controlled clinical trials included in systematic reviews. This included quantitative, qualitative, graphical or tabular techniques, suggestions or methods. 


\section{Exclusion criteria}

Systematic reviews of interventions for efficacy were excluded.

\section{Data extraction}

A data extraction form was developed and piloted independently by two individuals (JG, DM) on a random selection of 10 included resources. Extractions were checked for consensus and the form revised according to the feedback provided. One person extracted information for all included studies (JG) regarding why the authors sought to assess clinical heterogeneity; what "criteria" were used to assess clinical heterogeneity; how these were developed; the definition of clinical heterogeneity used by the authors; any graphical, tabular or other display/summary methods; statistical recommendations; reported methods used; empirical validation performed on the "criteria"; examples of implementing the methods; and recommendations on how the assessments are to be used in systematic reviews. All extractions were checked for accuracy by another individual (DM).

\section{Synthesis methods}

We thematically grouped the retrieved resources, suggestions or techniques (e.g., statistical versus qualitative recommendations), described the recommendations, highlighted any empirical support cited for each recommendation, and made an overall summary of the recommendations.

\section{Assessment of method validation}

Four individuals (JG, DM, JB, CB) met several times to discuss how to rate the variety of resources retrieved. These individuals came to a consensus that there were several classes of resources that did not have any accepted risk-of-bias assessment tools or instruments (e.g., textbooks, narrative reviews, learning guides, expert opinions). Therefore instead, of assessing "risk of bias" of these articles, we chose to determine if specific methods have been validated. Resources were considered validated if they had a clear rationale or reported empirical evidence for that recommendation (e.g., reference to previous empirical work or a test of the method with empirical or simulated data). One individual (JG) assessed the method of validation of each of these included resources.

\section{Results}

Our searches identified 2497 unique titles and abstracts; after screening, 101 papers were included in the review [17-117]. These resources included statistical papers,
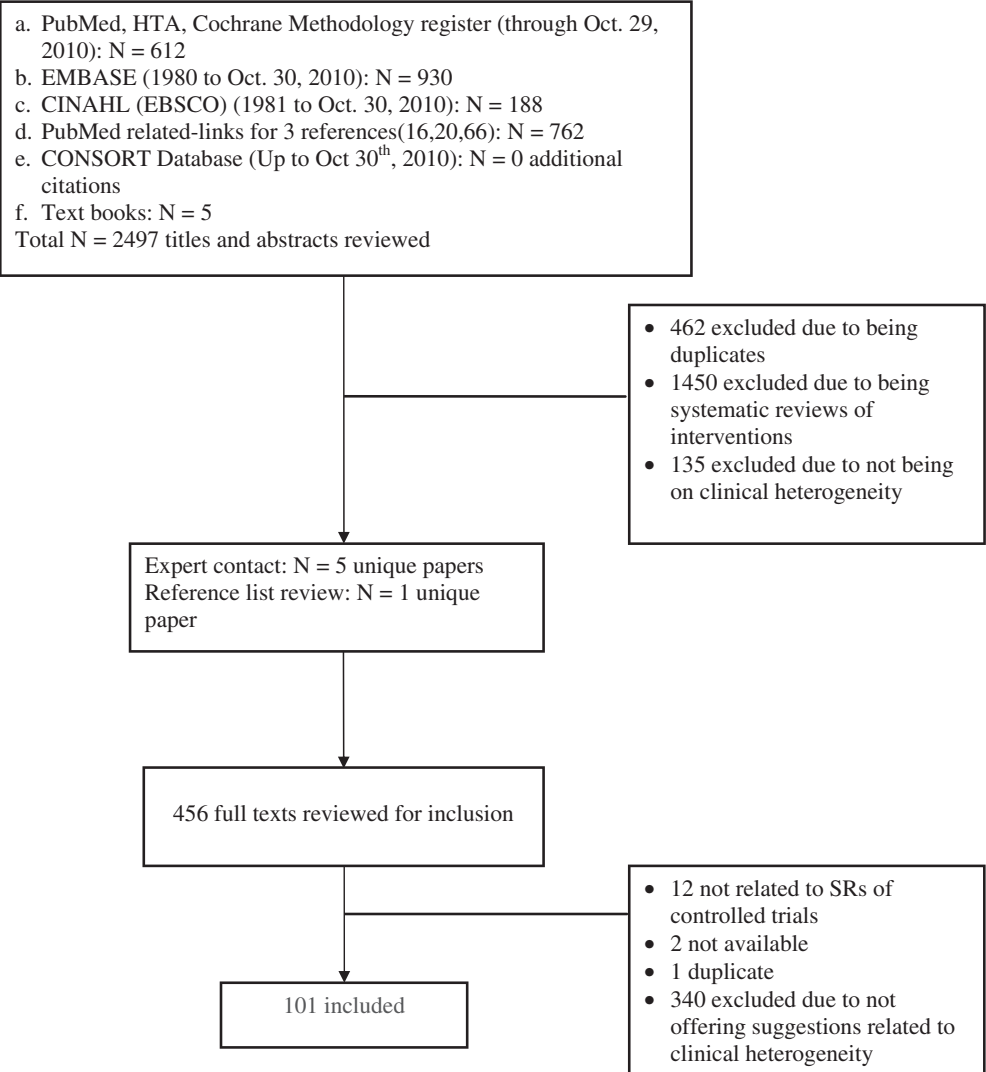

Figure 1 Search and inclusion results. 
methodological reviews, narrative reviews, expert opinions, learning guides, consensus-based guidelines and textbooks. Figure 1 describes details of the search and screening results. The very few disagreements on inclusion were easily resolved through discussion. Sixty-four (64.6\%) of the resources (statistical, methodological, consensus guideline resources) were assessed for validation. Forty-one (64.1\%) of these references were evaluated as being sufficiently validated.

Table 1 describes some basic characteristics of the included resources. The most common type of resource was statistical papers (42.4\%), with narrative reviews/expert opinion papers being the next most common (29.3\%). Most of the papers were published in the 2000s (70.1\%), and statistical methods for investigating clinical heterogeneity were the most frequent types of suggestions across resources (73.7\%). Table 2 reports a list of clinical variables suggested for investigating clinical heterogeneity and the number and types of resources suggesting each. General suggestions of clinically related variables, without identification of specific clinical covariates, were the most common across all included resources. Most suggestions were within distinct categories: participant level (e.g., age), intervention level (e.g., dose), or outcome level (e.g., event type, length of follow-up) covariates. A number of resources $(\mathrm{N}=14)$ reported control event rate/baseline risk as being a covariate worth investigating.

Table 1 Descriptive characteristics of included resources that reported recommendations for investigating clinical heterogeneity in systematic reviews of controlled clinical trials $(N=101)$

\begin{tabular}{llr}
\hline Descriptive Characteristics & $\mathbf{N}^{\mathbf{1}}$ \\
\hline Type of publication & $\begin{array}{l}\text { Statistical paper } \\
\text { Narrative review or } \\
\text { expert opinion }\end{array}$ & 44 \\
& Methodological review & 29 \\
& Consensus-based guideline & 14 \\
& Textbook & 9 \\
& 2000s & 5 \\
Decade of publication & 1990s & 70 \\
& 1980s & 27 \\
$\begin{array}{l}\text { Guidance on statistical } \\
\text { methods }\end{array}$ & & 75 \\
$\begin{array}{l}\text { Clinical variables } \\
\text { (general }{ }^{2} \text { or specific) } \\
\text { recommended }\end{array}$ & & 39 \\
$\begin{array}{l}\text { Process for choosing } \\
\text { clinical variables recommended }\end{array}$ & 28 \\
\hline
\end{tabular}

1. The number $(\mathrm{N})$ of resources equals the percentage of resources since we include 101 total resources.

2. The term "general" means that the resource listed the term "patient", "intervention", or "outcome" as a category from which to consider covariates without suggesting specific variables.
Table 2 Types of clinical covariates suggested across all resources

\begin{tabular}{lll}
\hline General Category & $\begin{array}{l}\text { Specific } \\
\text { Covariate }\end{array}$ & $\begin{array}{l}\text { Number of } \\
\text { Resources } \\
\text { Recommending }\end{array}$ \\
\hline Patient level & General $^{2}$ & 15 \\
& Age & 7 \\
& Baseline severity & 3 \\
& Sex/gender & 4 \\
& Ethnicity & 2 \\
& Comorbidities & 2 \\
& Other disease features & 2
\end{tabular}

Intervention level General 13

Dose 8

Duration 5

Brand 3

Co-interventions 3

Intensity 3

Timing 3

Route 2

Compliance 2

Others unique to the 2 intervention

Frequency 1

Comparator/control 1

Outcome level General 6

Event type 5

Length of follow-up $\quad 4$

Outcome measure type 3

Outcome definition 3

Timing 2

Repeated outcome $\quad 1$

Control event rate / 14

baseline risk

Research setting 4

Comparison conditions 3

Early stopping rules 1

Population risk 1

1. The number $(\mathrm{N})$ of resources equals the percentage of resources since we include 101 total resources.

2. The term "general" means that the resource listed the term "patient", "intervention", or "outcome" as a category from which to consider covariates without suggesting specific variables.

Table 3 lists recommendations regarding the process of choosing clinical characteristics to investigate. Five or more resources suggested the following: a priori choice of clinical covariates (e.g., in the review protocol); look at forest plots for trials that may contribute to heterogeneity and then look for clinical characteristics therein; 
Table 3 Recommendations regarding the methods of choosing or identifying clinical covariates for investigation and interpretation of the findings

\begin{tabular}{|c|c|c|c|}
\hline $\begin{array}{l}\text { General Category of } \\
\text { Recommendation }\end{array}$ & Specific Recommendation & $\begin{array}{l}\text { Number of } \\
\text { Resources }\end{array}$ & Citations \\
\hline $\begin{array}{l}\text { When to identify covariates } \\
\text { in the review process }\end{array}$ & A priori (e.g., in protocol) & 17 & $\begin{array}{l}76,92,93,95,100,98,18,26,39 \\
40,30,59,29,31,46,94,114\end{array}$ \\
\hline \multirow[t]{17}{*}{$\begin{array}{l}\text { How to find important } \\
\text { clinical covariates from } \\
\text { trial information }\end{array}$} & $\begin{array}{l}\text { Looking at forest plots } \\
\text { (variation in point estimates/Cl } \\
\text { overlap/ adding a } \\
\text { vertical line for levels of } \\
\text { some clinical variable) }\end{array}$ & 6 & $92,98,93,97,98,94$ \\
\hline & $\begin{array}{l}\text { Proceed regardless of formal } \\
\text { testing of statistical } \\
\text { heterogeneity }\end{array}$ & 5 & $35,92,97,98,29$ \\
\hline & Looking at L'Abbe plots & 4 & $98,45,93,98$ \\
\hline & Influence plot & 3 & $98,54,85$ \\
\hline & Looking at summary tables & 2 & 92,24 \\
\hline & Looking at funnel plots & 2 & 49,98 \\
\hline & $\begin{array}{l}\text { Use conceptual frameworks to } \\
\text { facilitate choice of covariates } \\
\text { (i.e., using taxonomies for } \\
\text { active ingredients) }\end{array}$ & 2 & 98,112 \\
\hline & $\begin{array}{l}\mathrm{I}^{2} \text { (look at the change in } \\
\text { statistical heterogeneity by } \\
\text { adding subgroups) }\end{array}$ & 2 & 87,100 \\
\hline & $\begin{array}{l}\text { Plot of effect size against } \\
\text { individual covariates }\end{array}$ & 1 & 48 \\
\hline & $\begin{array}{l}\text { Using an adaptation of } \\
\text { multidimensional } \\
\text { scaling (CoPlot) }\end{array}$ & 1 & 55 \\
\hline & Plot of normalized z-scores & 1 & 93 \\
\hline & Radial/Galbraith plot & 1 & 93 \\
\hline & Frequency distributions & 1 & 98 \\
\hline & Dose-response graph & 1 & $3 ?$ \\
\hline & $\begin{array}{l}\text { Use P.I.C.O. model to guide } \\
\text { choice of characteristics }\end{array}$ & 1 & 115 \\
\hline & $\begin{array}{l}\text { Use causal mediating } \\
\text { processes }\end{array}$ & 1 & 113 \\
\hline & $\begin{array}{l}\text { Treat strata within trials as } \\
\text { separate studies; these } \\
\text { subgroups if similar across } \\
\text { studies can be combined }\end{array}$ & 1 & 46 \\
\hline \multirow[t]{4}{*}{$\begin{array}{l}\text { Rationale for choice } \\
\text { of covariate }\end{array}$} & $\begin{array}{l}\text { Scientific (e.g., } \\
\text { pathophysiological, } \\
\text { pharmacologic argument) }\end{array}$ & 10 & $7,76,92,93,100,18,26,59,31,115$ \\
\hline & $\begin{array}{l}\text { Previous research } \\
\text { (e.g., large } \mathrm{RCT} \text { ) }\end{array}$ & 3 & $76,68,100$ \\
\hline & Clinical grounds & 2 & 96,100 \\
\hline & Indirect evidence & 1 & 59 \\
\hline \multirow[t]{2}{*}{ Personnel } & Use of clinical experts & 2 & 21,115 \\
\hline & Blind to results of trials & 1 & 35 \\
\hline $\begin{array}{l}\text { Number of } \\
\text { covariates/ } \\
\text { trials needed }\end{array}$ & Small number of covariates & 7 & $92,95,100,18,26,31,94$ \\
\hline
\end{tabular}


Table 3 Recommendations regarding the methods of choosing or identifying clinical covariates for investigation and interpretation of the findings (Continued)

\begin{tabular}{|c|c|c|c|}
\hline & $\begin{array}{l}\text { Each covariate investigation } \\
\text { should be based on an } \\
\text { adequate number of studies } \\
\text { (e.g., } 10 \text { for every moderator) }\end{array}$ & 6 & $100,59,50,94,115$ \\
\hline & $\begin{array}{l}\text { Investigators must report } \\
\text { actual number of covariates } \\
\text { investigated for reader to } \\
\text { determine the potential for } \\
\text { false-positives }\end{array}$ & 1 & 115 \\
\hline \multirow[t]{2}{*}{$\begin{array}{l}\text { Number of outcomes } \\
\text { to investigate }\end{array}$} & $\begin{array}{l}\text { Restrict investigations to small } \\
\text { number of outcomes } \\
\text { (e.g., primary) }\end{array}$ & 1 & 26 \\
\hline & $\begin{array}{l}\text { Limit to central question in } \\
\text { the analysis }\end{array}$ & 1 & 94 \\
\hline \multirow[t]{15}{*}{$\begin{array}{l}\text { Interpretation of results } \\
\text { of investigations }\end{array}$} & $\begin{array}{l}\text { Use caution ( } 4 \text { resources note } \\
\text { especially with post hoc } \\
\text { testing) }\end{array}$ & 12 & $100,18,29,31,85,16,20,23,25,61,32,35$ \\
\hline & Observational only & 6 & $59,23,94,98,100,114$ \\
\hline & $\begin{array}{l}\text { Exploratory or hypothesis } \\
\text { generating only }\end{array}$ & 4 & $32,100,40,94$ \\
\hline & $\begin{array}{l}\text { Consider confounding } \\
\text { between covariates }\end{array}$ & 4 & $100,50,115,59$ \\
\hline & $\begin{array}{l}\text { Consider artifactual causes of } \\
\text { between-study variation }\end{array}$ & 2 & 6,98 \\
\hline & $\begin{array}{l}\text { Consider biases (e.g., } \\
\text { misclassification, } \\
\text { dilution, selection) }\end{array}$ & 2 & 93,115 \\
\hline & $\begin{array}{l}\text { Look at magnitude of the } \\
\text { effect and the } 95 \% \mathrm{Cl} \text {; not just } \\
\text { effect and p-value; consider } \\
\text { precision of the subgroup } \\
\text { effects (e.g., sample sizes in } \\
\text { the studies dictate precision of } \\
\text { the subgroup effects) }\end{array}$ & 2 & 100,115 \\
\hline & $\begin{array}{l}\text { Seek evidence to justify claims } \\
\text { of subgroup findings }\end{array}$ & 1 & 26 \\
\hline & $\begin{array}{l}\text { Identify knowledge gaps in } \\
\text { the investigations }\end{array}$ & 1 & 24 \\
\hline & $\begin{array}{l}\text { Consider effect of variability } \\
\text { within studies }\end{array}$ & 1 & 19 \\
\hline & $\begin{array}{l}\text { Consider if the magnitude } \\
\text { is clinically important (i.e., } \\
\text { differences in effect between } \\
\text { subgroups) }\end{array}$ & 1 & 100 \\
\hline & $\begin{array}{l}\text { Think through causal } \\
\text { relationships, especially } \\
\text { directionality }\end{array}$ & 1 & 113 \\
\hline & $\begin{array}{l}\text { Use caution with variables } \\
\text { grouped after randomization }\end{array}$ & 1 & 23 \\
\hline & $\begin{array}{l}\text { Consider parabolic } \\
\text { relationships (i.e., beyond } \\
\text { linear regression) }\end{array}$ & 1 & 115 \\
\hline & $\begin{array}{l}\text { Be cautious not to say there is } \\
\text { a consistency of effect if no } \\
\text { subgroup effects are found }\end{array}$ & 1 & 115 \\
\hline
\end{tabular}




\begin{tabular}{|c|c|c|c|}
\hline \multirow[t]{2}{*}{ Descriptive methods } & $\begin{array}{l}\text { Perform a narrative synthesis } \\
\text { of these investigations }\end{array}$ & 4 & $115,98,27,100$ \\
\hline & $\begin{array}{l}\text { Other: } 1 . \text { idea webbing, } 2 . \\
\text { qualitative case descriptions, } 3 . \\
\text { investigator/methodological/ } \\
\text { conceptual triangulation }\end{array}$ & 1 & 98 \\
\hline \multirow[t]{4}{*}{ Use of types of data } & $\begin{array}{l}\text { Aggregate patient data for trial } \\
\text { level covariates }\end{array}$ & 4 & $23,25,118,46$ \\
\hline & $\begin{array}{l}\text { Only group characteristics } \\
\text { derived prior to randomization } \\
\text { (e.g., stratifying) }\end{array}$ & 2 & 23,46 \\
\hline & $\begin{array}{l}\text { Individual patient data for } \\
\text { participant level covariates }\end{array}$ & 1 & 59 \\
\hline & $\begin{array}{l}\text { Individual patient data only for } \\
\text { all covariates where possible }\end{array}$ & 1 & 59 \\
\hline
\end{tabular}

1. The number $(\mathrm{N})$ of resources equals the percentage of resources since we include 101 total resources.

proceed with investigation regardless of results of formal testing for statistical heterogeneity; base clinical covariates on a clear scientific rationale (e.g., a pathophysiological argument); investigate a small number of covariates; base each covariate suggestion on an adequate number of trials (e.g., 10 trials was a common suggestion); use caution when interpreting the findings of investigations; consider the results of such investigations as exploratory, hypothesis generating and observational; and consider confounding between covariates.

Table 4 summarizes the types of statistical methods suggested for investigating clinical heterogeneity characteristics and the number of resources suggesting each. Many included resources made some mention of statistical methods of investigating aspects of clinical heterogeneity $(\mathrm{N}=69 / 99,69.7 \%)$. Also, many of these resources made general suggestions regarding the use of subgroup analyses $(\mathrm{N}=18)$ and meta-regression $(\mathrm{N}=16)$; however, the majority of these did not offer any specific recommendations. A wide variety of meta-regression techniques were suggested, many of which included simulated evidence or other forms of empirical testing. Several Bayesian approaches were suggested as well as several methods for individual patient data analysis $[34,48,58,63,66,69,71,75,95]$. Four textbooks appeared to be relatively comprehensive in their treatment of statistical recommendations [93-95,114].

Overall, we felt that there was some consensus across the resources regarding planning investigations, the use of clinical expertise, the rationale for choice of covariate, how to think through types of covariates, making a covariate hierarchy, post hoc covariate identification, statistical methods, data sources and interpretation of findings (See Table 5). We summarize the common recommendations that appeared in the literature to offer some preliminary guidance for systematic reviewers in
Table 5 and we elaborate on several key areas in the discussion section below.

Sources appearing to be the most comprehensive in their discussion of recommendations for investigating clinical heterogeneity included the Cochrane Handbook [100] and the Centre for Reviews and Dissemination's Guidance For Undertaking Reviews In Health Care [98] and the AHRQ Comparative effectiveness review methods: clinical heterogeneity [115].

\section{Discussion}

A variety of decisions must be made when performing a systematic review. One such decision is how to deal with obvious differences among and within trials. Though a significant test for the presence of statistical heterogeneity (e.g., Q test) and a large degree of heterogeneity (e.g., $\mathrm{I}^{2}>75 \%$ ) might obligate a reviewer to look for covariates to explain this variability, a nonsignificant test or a small $\mathrm{I}^{2}$ (e.g., <25\%) does not preclude the need to investigate covariate treatment effect interactions $[35,92,97,100]$. That is, even with low statistical heterogeneity, there may still be factors that influence the size of the treatment effect, especially if there is a strong argument (i.e., pathophysiologic or otherwise) that some variable likely does have such an influence.

Observed or expected heterogeneity of treatment effects can be handled in several ways. The heterogeneity can be ignored and a meta-analysis conducted with a fixed-effects or random-effects model, or one can attempt to explain the heterogeneity through subgroup analyses, meta-regression or other techniques [25]. The latter moves the review away from overall statements of evidence to increasingly clinically applicable results and conclusions as well as new hypotheses for future research [75]. Anello and Fleiss [28] make a clear distinction between meta-analyses with a goal of arriving at a 
Table 4 Statistical suggestions for investigating aspects of clinical heterogeneity

\begin{tabular}{|c|c|}
\hline $\begin{array}{l}\text { General Category of } \\
\text { Statistical Method }\end{array}$ & Specific Method Suggested \\
\hline
\end{tabular}

Hierarchical testing procedure based on the heterogeneity statistic Q

Combining subgroups across studies (i.e., in stratified studies)

\section{Moderator Analyses}

1. ANOVA ${ }^{2}$ analogue

(e.g., a categorical moderator)

2. Meta-regression

General mention

Fixed effects model (general)

Bayesian models (general)

New maximum likelihood method

New weighted least squares model

Random effects model (general)

Random effects model for IPD ${ }^{3}$

Permutation-based resampling

Other nonparametric (e.g., fractional polynomials, splines)

Mixed effects model

New variance estimators (for covariates)

Methods for measurement of residual errors

Bayesian model in the presence of missing study-

level covariate data

Semi-parametric modeling (general)

Fixed effects generalized least squares model

Hierarchical regression models

Random effects model with new variance estimator

Logistic regression with binary outcomes

Interaction term for metaregression model

Consider nonlinear relationships (e.g., use quadratic or log transformations)

Bayesian model for use in meta-analyses of multiple treatment comparisons
$48,94,95,114$

$19,60,6,24,2528,31,32,43,50$,

$75,94,95,100,98,93,1325,418$

$92,93,94,95$

$66,71,124,95$

60, 124

58,67

67,114

58,61

31,43

69,85

38,114

77,84

59,41

110

80

68

$60,64,124$

70

25

95

48 
Table 4 Statistical suggestions for investigating aspects of clinical heterogeneity (Continued)

\author{
3. Multivariate analyses \\ 4. Multiple univariate analyses \\ with Bonferroni adjustments \\ 5. Meta-analysis of interaction \\ estimates \\ 6. Model to include the \\ repeated observations \\ (time as a variable) using IPD \\ 7. Z test
}

\section{Bayesian Approaches}

1. Hierarchical Bayesian

modeling

2. Random effects models

Data Specific Approaches

\author{
1. IPD analyses
}

2. Combination of IPD \& APD ${ }^{4}$

\section{Other Approaches}

Models for control event rate / baseline risk

Structural equation modeling (SEM)

Mixed treatment comparisons

combined with

meta-regression

Combining regression

coefficients from

separate studies

\begin{tabular}{|c|c|c|}
\hline & 1 & 48 \\
\hline & 1 & 48 \\
\hline & 1 & 61 \\
\hline & 1 & 109 \\
\hline & 1 & 125 \\
\hline & 2 & 44,48 \\
\hline & 1 & 63 \\
\hline General & 5 & $75,76,95,97,23$ \\
\hline Regression & 1 & 61,46 \\
\hline $\begin{array}{l}\text { Adding a treatment- } \\
\text { covariate interaction term }\end{array}$ & 1 & 95 \\
\hline Two-step models & 2 & 74,78 \\
\hline Multi-level model & 2 & 69,100 \\
\hline $\begin{array}{l}\text { Meta-analysis of } \\
\text { interaction estimates }\end{array}$ & 1 & 61 \\
\hline $\begin{array}{l}\text { General (e.g., control } \\
\text { event rate) }\end{array}$ & 10 & $63,24,71,81,79,93,100,19,78,111$ \\
\hline Integration of SEM with fixed, & 1 & 42 \\
\hline & 1 & 72 \\
\hline & 1 & 64 \\
\hline
\end{tabular}

1. The number $(\mathrm{N})$ of resources equals the percentage of resources since we include 101 total resources; 2 . ANOVA = analysis of variance; 3 . IPD =individual patient data; 4 . APD $=$ aggregate patient data.

common summary estimate of effect ("analytic metaanalyses") and those focused on explaining why the effect sizes vary ("exploratory or causal meta-analyses"). The choice between these depends on the objective of the review, but it is clear that meta-analyses are more applicable to decision making (e.g., clinical, policy) when they are exploratory in nature $[14,28,53,75,99]$. The trials included in a systematic review may be so very similar that the summary effect estimate is the most reasonable and applicable metric [114]. But these cases are very rare, and therefore we would expect most questions asked and tested through meta-analytic methods should concern possible reasons for variation in effect [114].

Many resources were found that suggested methods for carrying out investigations of clinical heterogeneity in systematic reviews [17-102]. There was great variety in the types of resources identified (statistical papers to commentaries) and in their potential for risk of bias. It was decided early to include any resource, no matter the design, methods, or publication type. For this reason many of the included resources might normally be considered at a high risk of bias (e.g., narrative reviews, expert opinions, learning guides and commentaries) and thus providing suggestions of questionable validity. But it was felt that these types of resources might provide the most valuable information on the subject of clinical heterogeneity. That is, investigating, and in particular choosing which clinical characteristics to investigate, requires clinical expertise, or at a minimum, knowledge of empirical evidence of some covariate of importance. The inclusion of these resources could be viewed as a drawback, but we saw it as a strength of this research. It 
Table 5 Summary of recommendations for investigating clinical heterogeneity in systematic reviews

\begin{tabular}{|c|c|}
\hline Recommendation Category & Recommendation Description \\
\hline A-priori planning & $\begin{array}{l}\text { 1. All plans for investigating clinical } \\
\text { heterogeneity should be made explicit, } \\
\text { a-priori (e.g., in the protocol for the } \\
\text { systematic review). }\end{array}$ \\
\hline Clinical expertise & $\begin{array}{l}\text { 2. The review/investigative team should } \\
\text { include clinical experts or state a plan } \\
\text { for consulting clinical experts during the } \\
\text { review protocol development and } \\
\text { implementation (e.g., when choosing } \\
\text { clinical covariates and when } \\
\text { interpreting the findings). }\end{array}$ \\
\hline
\end{tabular}

Covariate rationale $\quad 3$. Clinical covariates should be chosen that have a clearly stated rationale for their importance (e.g., a pathophysiological argument, reference to the results of a previous trial).

4. Review teams should think through
Thinking through covariate categories

Covariate hierarchy

Post hoc covariate identification

Statistical methods

Data sources

Interpretation the following categories to determine if related covariates might logically influence the treatment effect in their particular review: participant level, intervention level, outcome level, research setting, or others unique to their research question.

5. A logical hierarchy of clinical covariates should be formed and investigated only if there is sufficient rationale and a sufficient number of trials available (10 trials per covariate).

6. State any plans to include additional covariates after looking at the data (post hoc) from included studies (e.g., forest plots, radial plots) and how they plan to do this.

7. Describe a-priori the statistical methods proposed to investigate identified covariates. Refer to accepted texts or published papers in the area to be sure to implement these methods in a valid manner. Include an individual with experience in conducting these analyses. ${ }^{1}$

8. Aggregate patient data: Reasonable for investigating trial level covariates

9. Individual patient data: Consider when investigating participant level covariates (otherwise results are subject to ecologic bias)

10. A. Protocol: Describe how the results of any findings are going to be interpreted and used in the overall synthesis of evidence.

B. Review: Consider the observational nature of these investigations; consider confounds and important potential biases; consider magnitude of the effect, confidence intervals and directionality of the effect.

\footnotetext{
${ }^{1}$ We do not provide detailed recommendations for statistical analyses here because of the breath and complexity of this topic. Instead we suggest that one refer to accepted resources and well-trained individuals with expertise in the area.
}

was these resources that provided most of the suggestions regarding the methods for choosing or identifying clinical covariates to investigate (Table 3). The consensus-based guidelines provided most of the suggestions regarding the process of choosing or identifying clinical covariates, and the statistical papers, as might be expected, covered the majority of the specific statistical suggestions; but the textbooks also offered many suggestions in both areas. There was some consensus across resources, but only a small number of resources included a relatively comprehensive set of recommendations $[15,93,94,98]$. Therefore, future research should be directed at developing a comprehensive and up to date set of guidelines to aid reviewers in investigating clinical heterogeneity. We summarize the common recommendations that appear in the literature to offer some preliminary guidance for systematic reviewers (Table 5).

We were surprised to see that the term clinical heterogeneity was relatively commonly used and consistently defined. We took our definition from several publications with which we were previously familiar $[1,3]$. In some of the resources the term methodological heterogeneity was used synonymously with clinical heterogeneity, or clinical heterogeneity was considered to be one component of methodological heterogeneity. While this was infrequent in the literature, methodological aspects of heterogeneity include but go beyond clinical aspects or reasons for heterogeneity between trials. Thus, when describing reasons for heterogeneity that are related to the participants, intervention, outcomes or settings of the trial, these should be termed clinical aspects of heterogeneity. A consistency of terminology is mandatory for development of thought and investigation in this area. With terminology in place, the discussion can move to our recommendations.

When planning investigations of clinical heterogeneity in systematic reviews of controlled trials one should make such plans explicit, a priori, in the protocol for the review. We would suggest that protocols be published or registered in appropriate databases [118]. Next, it is reasonable and arguably beneficial, when organizing the review team, to include clinical experts or at a minimum, state a plan for consulting clinical experts during particular phases of the review (e.g., when choosing clinical covariates or during interpretation of findings). Furthermore, a set of clinical covariates should be chosen that have a clearly stated rationale for their importance (e.g., pathophysiological argument or reference to the results of a previous large trial). Review teams should think through the following categories to determine if related covariates might logically influence the treatment effect in their particular review: participant level, intervention level, outcome level, research setting, or others unique to the research question. Several resources offered 
conceptual mapping, idea webbing and causal modeling as possible methods for identifying important covariates and relationships between them [98,112,113]. Next, a hierarchy of clinical covariates should be formed and covariates investigated only if there is sufficient rationale and later a sufficient number of trials available. That is, covariates deemed more important than others on the basis of an explicitly stated rationale should be immediately included in such investigations, with other covariates being included when the number of trials is sufficient. A generally accepted rule of thumb is that 10 events per predictor variable (EPV) maintains bias and variability at acceptable levels. This rule derives from 2 simulation studies carried out for logistic and Cox modeling strategies [119-121] and has been adapted to metaregression $[1,114]$. Therefore, it has been suggested that for each covariate there should be at least 10 trials to avoid potentially spurious findings [15]. Also, investigators should describe any plans to include additional covariates after looking at the data from included studies (e.g., forest plots). This might include an examination of summary tables or various types of plots $[92,93,97,98,106]$, and it would be reasonable to include the clinical expert(s) at this stage to aid in the interpretation of the plotted data. Finally, how the results of any findings are going to be interpreted and used in the synthesis methods of the review needs to be explained. Most resources advise caution in interpreting these investigations, noting their exploratory nature, but when there is a clearly stated rationale, especially when derived from previous research, and sufficient trials are included, a priori planned investigations may improve applicability. Also, it was frequently suggested that the interpretation of the results of these investigations should consider confounds and important potential biases, the magnitude of the effect, confidence intervals and the directionality of the effect. Following these recommendations may lead to valid and reliable investigations of clinical heterogeneity and could improve their overall applicability and lead to future research that might test hypothesized subgroup effects.

A wide variety of statistical analyses are available for investigating clinical heterogeneity in systematic reviews of controlled clinical trials, and it is not within the scope of this paper to cover these in detail. Other resources cover this subject very well $[15,93,95,100,114]$. The sophistication of techniques is constantly growing, and an updated, precise summary of such methods is needed. Instead we will describe three available options frequently suggested by resources included in our review-subgroup analyses, meta-regression and the analogue to the analysis of variance (ANOVA) - and comment upon methods for exploring control group event rate.
Subgroup analyses involve separating trials into groups on the basis of some characteristic (e.g., intervention dose) and then performing separate meta-analyses for each group. This test provides an effect estimate within subgroups and a significance test for that estimate; it does not provide a test of variation in effect due to covariates. The greater the number of significant tests performed, the greater the likelihood of type 1 errors. There are some suggestions in the literature for how to control for this (e.g., Bonferroni adjustments [48]). To test for differences between subgroups a moderator analysis must be done. Moderator analyses include metaregression and the analogue to the ANOVA, among other techniques (e.g., Z test [114]). Meta-regression is used to assess the impact of one or more independent variables (e.g., age or intervention dose) upon the dependent variable, the overall treatment effect [62]. Independent variables may be continuous or categorical, the latter expressed as a set of dummy variables with one omitted category. Several modeling strategies are available for performing meta-regression $[100,108,122]$. The results of meta-regression indicate which variables influence the summary treatment effect, how much the summary effect changes with each unit change in the variable and the $\mathrm{p}$-value of this influence. It has been suggested that at least 10 trials per covariate are needed to limit spurious findings, due to the low statistical power of meta-regression, and a nonparametric test has been suggested when this tenet is not fulfilled [30] Also, one needs to consider the problems associated with ecological bias when performing meta-regressions on patient levels variables [40]. Finally, the analogue to the ANOVA examines the difference in the effect between categorical levels of some variable using identical statistical methods as a standard ANOVA [94].

The literature suggests many methods for examining the influence of the control event rate or baseline risk, which is considered an aggregate measure of known (e.g., age and disease severity) and unknown variables $[15,43,93]$. It has been argued that these examinations provide little import to clinical practice since the influence of any possible causative variables is aggregated and therefore the effect of individual covariates is unknown [15]. Also, the influence of the control event rate on the summary affect is affected by regression to the mean, and sophisticated statistical procedures are required to deal with this $[15,43,93]$.

Bayesian approaches to meta-regression and hierarchical Bayes modeling, among other areas, appear to be well represented in the literature $[66,71,95]$, as well as more general resources for Bayesian meta-analytic techniques $[95,123]$. These methods are developing rapidly; therefore, frequent summaries of these important techniques are required as a resource to reviewers. 
Finally, we would like to note suggestions in the literature concerning the utility of aggregate patient data (APD) versus individual patient data (IPD). Several resources give general recommendations regarding use of IPD when exploring characteristics that could be considered aspects of clinical heterogeneity [15,74-76,95,97]. Some empirical evidence supports these recommendations $[40,66,124,125]$. When IPD is available, it should be used as a basis to investigate aspects of clinical heterogeneity at the patient level (e.g., demographic characteristics) so as to avoid ecological bias associated with summary APD. It is reasonable to use APD for trial-level covariates (e.g., intervention characteristics) that can be considered aspects of clinical heterogeneity. In addition, there may be opportunities to strategically use APD together with IPD to avoid the significant, and sometimes insurmountable, effort required to collect complete IPD [71].

Finally, in relation to the suggestions above for including clinical expertise in systematic reviews, we feel it is the responsibility of each therapeutic discipline to create a repository of variables to consider when exploring effect variation in systematic reviews. Such warehousing of clinically important covariates would serve as an important resource, allowing systematic reviewers and clinical trialists to explore nuances in treatment effect that might inform clinical decision making, and allowing for increased applicability of findings.

\section{Conclusions}

In summary, although many recommendations are available for investigating clinical heterogeneity in systematic reviews of controlled clinical trials, there is a need to develop a comprehensive set of recommendations for how to perform valid, applicable, and appropriate investigations of clinical covariates $[7,14]$. This will improve the applicability and utilization of systematic reviews by policy makers, clinicians, and other decision makers and researchers who wish to build on these findings.

\section{Appendix A: Search strategies \\ 1. OVID searches}

Medline (1950 to Oct $\left.29^{\text {th }}, 2010\right)$; Cochrane Methodology Register (Oct 29 $\left.{ }^{\text {th }}, 2010\right)$; HTA (Oct 29 $9^{\text {th }}, 2010$ ); EMBASE (1980 to Oct $\left.30^{\text {th }}, 2010\right)$

"(((clinical adj5 heterogeneity)) and (assessment or checklist or guideline or guidelines or scale or criteria))"

Note: A slight variation in this strategy was used for EMBASE, on the EMBASE specific search engine, for an updated search we performed from January $1^{\text {st }} 2009$ to October $30^{\text {th }}, 2010$. This was due to a change in the available electronic resources.
2. CINAHL (EBSCO) (1981 up to October $\left.30^{\text {th }}, 2010\right)$ :

“TX clinical N8 heterogeneity and TX ( assessment OR checklist OR guideline OR guidelines OR scale OR criteria )"

\section{CONSORT database of methodological papers (up to Oct $30^{\text {th }}, 2010$ )}

Manual search of all citations.

\section{Related PubMed links for (completed on October $31^{\text {st }}$, 2010):}

Thompson SG. Why sources of heterogeneity in metaanalysis should be investigated. BMJ. 1994;309:1351-5.

\section{Related PubMed links for (completed on October $31^{\text {st }}$, 2010):}

Higgins J, Thompson S, Deeks J, Altman D. Statistical heterogeneity in systematic reviews of clinical trials: a critical appraisal of guidelines and practice. Journal of Health Services \& Research Policy. Jan 2002;7(1):51-61.

\section{Related PubMed links for: (completed on October $31^{\text {st }}$, 2010)}

Schmid CH, Stark PC, Berlin JA, Landais P and Lau J. Meta-regression detected associations between heterogeneous treatment effects and study-level, but not patient-level, factors. Journal of Clinical Epidemiology. 2004;57:683-97.

\section{Competing interests}

The authors declare that they have no competing interests.

\section{Authors' contributions}

JG developed conceptualized the project, searched for the literature, extracted data, and wrote the manuscript. DM, HB, JB and CB conceptualized the project and edited the manuscript. All authors read and approved the final manuscript.

\section{Author details}

'Departments of Orthopaedic Surgery and Epidemiology, University of Michigan, 24 Frank Lloyd Wright Drive, Ann Arbor, MI, USA. ${ }^{2}$ Clinical Epidemiology Program, Ottawa Health Research Institute, Ottawa, ON, Canada. ${ }^{3}$ Department of Epidemiology \& Community Medicine, Faculty of Medicine, University of Ottawa, Ottawa, ON, Canada. ${ }^{4}$ Leslie Dan Faculty of Pharmacy, University of Toronto, Toronto, ON, Canada. ${ }^{5}$ Child Health and Evaluative Sciences, The Hospital for Sick Children, Toronto, ON, Canada. ${ }^{6}$ Health Policy, Management \& Evaluation, Faculty of Medicine, University of Toronto, Toronto, ON, Canada.

Received: 6 September 2011 Accepted: 15 June 2012 Published: 30 July 2012

\section{References}

1. In Cochrane Handbook for Systematic Reviews of Interventions. Version 5.0.1 [updated September 2008]. Edited by Higgins JPT, Green S.: The Cochrane Collaboration; 2008. Available from www.cochrane-handbook.org.

2. Pildal J, Hrobjartsson A, Jorgensen KJ, Hilden J, Altman DG, Gotzsche PC: Impact of allocation concealment on conclusions drawn from metaanalyses of randomized trials. Int J Epidemiol 2007, 36(4):847-857.

3. Tugwell P, Robinson V, Grimshaw J, Santesso N: Systematic reviews and knowledge translation. Bulletin of the World Health Organization 2006, 84:643-651. 
4. Grimshaw JM, Santesso N, Cumpston M, Mayhew A, McGowan J: Knowledge for knowledge translation: the role of the cochrane collaboration. J Contin Educ Health Prof 2006, 26:55-62.

5. British Medical Journal, Clinical Evidence; 2009. Available at: http:// clinicalevidence.bmj.com/ceweb/about/index.jsp. Accessed February 16, 2009.

6. Canadian Institutes of Health Research. Available at: http://www.cihr-irsc.gc.ca/e/ 193.html. Accessed February 16, 2009

7. Cochrane Collaboration, Current News; Available at: http://cochrane.org/ archives/channel_2.htm. Accessed February 16, 2009.

8. Laupacis A, Strauss S, Systematic reviews: Time to address clinical and policy relevance as well as methodological rigor. Ann Int Med 2007, 147(4):273-275.

9. Moher D, Liberati A, Tetzlaff J, Altman DG, The PRISMA Group: Preferred reporting items for systematic review and meta-analyses: the PRISMA statement. PLoS Medicine 2009, 6(\&):e1000097.

10. Glasziou P, Meats E, Heneghan C, Shepperd S: What is missing from descriptions of treatment in trials and reviews? BMJ 2008, 336:1472-1474.

11. Glasziou P, Chalmers I, Altman DG, et al: Taking healthcare interventions from trial to practice. BMJ 2010, 341:384-387.

12. Chalmers I, Glasziou P: Avoidable waste in the production and reporting of research evidence. Lancet 2009, 374:86-89.

13. Chalmers I, Glasziou P: Avoidable waste in the production and reporting of research evidence. Obstet Gynecol 2009, 114(6):1341-1345.

14. Scott NA, Moga C, Barton P, Rashiq S, Schopflocher D, Taenzer P, Alberta Ambassador Program Team, et al: Creating clinically relevant knowledge from systematic reviews: The challenges of knowledge translation. J Eval Clin Pract 2007, 13(4):681-688.

15. Gagnier JJ, Bombardier C, Boon H, Moher D, Beyene J: An empirical study using permutation-based resampling in meta-regression. Systematic Reviews 2012, 1:18

16. Gagnier JJ, Morgenstern H, Moher D: Recommendations for investigating clinical heterogeneity in systematic reviews and meta-analyses. 2012, Under Review.

17. Arends LR, Hoes AW, Lubsen J, Grobbee DE, Stijnen T: Baseline risk as predictor of treatment benefit: Three clinical meta-re-analyses. Stat Med 2000, 19:3497-3518.

18. Higgins J, Thompson S, Deeks J, Altman D: Statistical heterogeneity in systematic reviews of clinical trials: a critical appraisal of guidelines and practice. J Health Serv Res Policy 2002, 7(1):51-61.

19. Schmid CH, Lau J, Mclntosh MW, Cappelleri JC: An empirical study of the effect of the control rate as a predictor of treatment efficacy in meta-analysis of clinical trials. Stat Med 1998, 17(17):1923-1942.

20. Thompson SG: Why sources of heterogeneity in meta-analysis should be investigated. BMJ 1994, 309(6965):1351-1355.

21. van den Ende CHM, Steultjens EMJ, Bouter LM, Dekker J: Clinical heterogeneity was a common problem in Cochrane reviews of physiotherapy and occupational therapy. J Clin Epidemiol 2006, 59:914-919.

22. Loke YK, Price D, Herxheimer A: Systematic reviews of adverse effects: framework for a structured approach. BMC Med Res Methodol 2007, 7:32

23. Freemantle N, Mason J, Eccles M: Deriving treatment recommendations from evidence within randomized trials. The role and limitation of meta-analysis. Int J Technol Assess Health Care 1999, 15(2):304-315.

24. Huang JQ, Zheng GF, Irvine EJ, Karlberg J: Assessing heterogeneity in meta-analyses of Helicobacter pylori infection-related clinical studies: a critical appraisal. Chin J Dig Dis 2004, 5(3):126-133.

25. Lau J, loannidis JP, Schmid CH: Quantitative synthesis in systematic reviews. Ann Intern Med 1997, 127(9):820-826.

26. Bender R, Bunce C, Clarke M, et al: Attention should be given to multiplicity issues in systematic reviews. J Clin Epidemiol 2008, 61(9):857-865

27. van Tulder M, Furlan A, Bombardier C, Bouter L: Updated method guidelines for systematic reviews in the Cochrane collaboration back review group. Spine 2003, 28(12):1290-1299.

28. Anello C, Fleiss JL: Exploratory or analytic meta-analysis: should we distinguish between them? J Clin Epidemiol 1995, 48(1):109-116. discussion 117-108.

29. Simmonds MC, Higgins JP, Stewart LA, Tierney JF, Clarke MJ, Thompson SG: Meta-analysis of individual patient data from randomized trials: a review of methods used in practice. Clin Trials 2005, 2(3):209-217.
30. Maxwell L, Santesso N, Tugwell PS, Wells GA, Judd M, Buchbinder R: Method guidelines for Cochrane Musculoskeletal Group systematic reviews. J Rheumatol 2006, 33(11):2304-2311.

31. Higgins JP, Thompson SG: Controlling the risk of spurious findings from meta-regression. Stat Med 2004, 23(11):1663-1682.

32. Song F, Sheldon TA, Sutton AJ, Abrams KR, Jones DR: Methods for exploring heterogeneity in meta-analysis. Eval Health Prof 2001, 24(2):126-151.

33. Glenton C, Underland V, Kho M, Pennick V, Oxman AD: Summaries of findings, descriptions of interventions, and information about adverse effects would make reviews more informative. J Clin Epidemiol 2006, 59(8):770-778.

34. Dohoo I, Stryhn H, Sanchez J: Evaluation of underlying risk as a source of heterogeneity in meta-analyses: a simulation study of Bayesian and frequentist implementations of three models. Prev Vet Med 2007, 81(1-3):38-55

35. Hall JA, Rosenthal R: Interpreting and evaluating meta-analysis. Eval Health Prof 1995, 18(4):393-407.

36. Gerbarg ZB, Horwitz RI: Resolving conflicting clinical trials: guidelines for meta-analysis. J Clin Epidemiol 1988, 41(5):503-509.

37. DerSimonian R, Laird N: Meta-analysis in clinical trials. Control Clin Trials 1986, 7(3):177-188.

38. St-Pierre NR: Invited review: Integrating quantitative findings from multiple studies using mixed model methodology. J Dairy Sci 2001, 84(4):741-755

39. Cook DJ, Sackett DL, Spitzer WO: Methodologic guidelines for systematic reviews of randomized control trials in health care from the Potsdam Consultation on Meta-Analysis. J Clin Epidemiol 1995, 48(1):167-171

40. Berlin JA, Santanna J, Schmid CH, Szczech LA, Feldman HI: Individual patient- versus group-level data meta-regressions for the investigation of treatment effect modifiers: ecological bias rears its ugly head. Stat Med 2002, 21(3):371-387.

41. Walter SD: Variation in baseline risk as an explanation of heterogeneity in meta-analysis. Stat Med 1997, 16(24):2883-2900.

42. Cheung MW: A model for integrating fixed-, random-, and mixed-effects meta-analyses into structural equation modeling. Psychol Methods 2008, 13(3):182-202.

43. Sutton AJ, Abrams KR, Jones DR, Sheldon TA, Song F: Systematic reviews of trials and other studies. Health Technol Assess 1998, 2(19):1-276.

44. Rosenthal R, DiMatteo MR: Meta-analysis: recent developments in quantitative methods for literature reviews. Annu Rev Psychol 2001 52:59-82.

45. Song F: Exploring heterogeneity in meta-analysis: is the L'Abbe plot useful? J Clin Epidemiol 1999, 52(8):725-730.

46. Reade MC, Delaney A, Bailey MJ, Angus DC: Bench-to-bedside review: Avoiding pitfalls in critical care meta-analysis-funnel plots, risk estimates, types of heterogeneity, baseline risk and the ecologic fallacy. Crit Care 2008, 12(4):220.

47. Xu H, Platt RW, Luo ZC, Wei S, Fraser WD: Exploring heterogeneity in meta-analyses: needs, resources and challenges. Paediatr Perinat Epidemiol 2008, 22(Suppl 1):18-28.

48. Olkin I: Diagnostic statistical procedures in medical meta-analyses. Stat Med 1999, 18(17-18):2331-2341

49. Sterne JA, Egger M, Smith GD: Systematic reviews in health care: Investigating and dealing with publication and other biases in metaanalysis. BMJ 2001, 323(7304):101-105.

50. Lipsey MW, Wilson DB: The way in which intervention studies have "personality" and why it is important to meta-analysis. Eval Health Prof 2001, 24(3):236-254.

51. Moher D, Jadad AR, Klassen TP: Guides for reading and interpreting systematic reviews: III. How did the authors synthesize the data and make their conclusions? Arch Pediatr Adolesc Med 1998, 152(9):915-920.

52. Schmid JE, Koch GG, LaVange LM: An overview of statistical issues and methods of meta-analysis. J Biopharm Stat 1991, 1(1):103-120.

53. Berlin JA: Invited commentary: benefits of heterogeneity in metaanalysis of data from epidemiologic studies. Am J Epidemiol 1995, 142(4):383-387.

54. Malling $H J$, Thomsen $A B$, Andersen JS: Heterogeneity can impair the results of Cochrane meta-analyses despite accordance with statistical guidelines. Allergy 2008, 63(12):1643-1645. 
55. Bravata DM, Shojania KG, Olkin I, Raveh A: CoPlot: a tool for visualizing multivariate data in medicine. Stat Med 2008, 27(12):2234-2247.

56. Baujat B, Mahe C, Pignon JP, Hill C: A graphical method for exploring heterogeneity in meta-analyses: application to a meta-analysis of 65 trials. Stat Med 2002, 21(18):2641-2652.

57. Higgins JP, Whitehead A: Borrowing strength from external trials in a meta-analysis. Stat Med 1996, 15(24):2733-2749.

58. Michiels S, Baujat B, Mahe C, Sargent DJ, Pignon JP: Random effects survival models gave a better understanding of heterogeneity in individual patient data meta-analyses. J Clin Epidemiol 2005, 58(3):238-245.

59. Thompson SG, Sharp SJ: Explaining heterogeneity in meta-analysis: a comparison of methods. Stat Med 1999, 18(20):2693-2708.

60. Smith CT, Williamson PR, Marson AG: Investigating heterogeneity in an individual patient data meta-analysis of time to event outcomes. Stat Med 2005, 24(9):1307-1319.

61. Simmonds MC, Higgins JP: Covariate heterogeneity in meta-analysis: criteria for deciding between meta-regression and individual patient data. Stat Med 2007, 26(15):2982-2999.

62. Thompson SG, Higgins JP: How should meta-regression analyses be undertaken and interpreted? Stat Med 2002, 21(11):1559-1573.

63. Thompson SG, Smith TC, Sharp SJ: Investigating underlying risk as a source of heterogeneity in meta-analysis. Stat Med 1997. 16(23):2741-2758

64. Frost $\mathrm{C}$, Clarke $\mathrm{R}$, Beacon $\mathrm{H}$ : Use of hierarchical models for meta-analysis: experience in the metabolic ward studies of diet and blood cholesterol. Stat Med 1999, 18(13):1657-1676.

65. Naylor CD: Two cheers for meta-analysis: problems and opportunities in aggregating results of clinical trials. Cmaj 1988, 138(10):891-895.

66. Schmid CH, Stark PC, Berlin JA, Landais P, Lau J: Meta-regression detected associations between heterogeneous treatment effects and study-level, but not patient-level, factors. J Clin Epidemio/ 2004, 57(7):683-697.

67. Higgins JP, Whitehead A, Turner RM, Omar RZ, Thompson SG: Meta-analysis of continuous outcome data from individual patients. Stat Med 2001, 20(15):2219-2241

68. Berkey CS, Anderson JJ, Hoaglin DC: Multiple-outcome meta-analysis of clinical trials. Stat Med 1996, 15(5):537-557.

69. Thompson SG, Turner RM, Warn DE: Multilevel models for meta-analysis, and their application to absolute risk differences. Stat Methods Med Res 2001, 10(6):375-392.

70. Berkey CS, Hoaglin DC, Mosteller F, Colditz GA: A random-effects regression model for meta-analysis. Stat Med 1995, 14(4):395-411.

71. Warn DE, Thompson SG, Spiegelhalter DJ: Bayesian random effects meta-analysis of trials with binary outcomes: methods for the absolute risk difference and relative risk scales. Stat Med 2002, 21(11):1601-1623.

72. Nixon RM, Bansback N, Brennan A: Using mixed treatment comparisons and meta-regression to perform indirect comparisons to estimate the efficacy of biologic treatments in rheumatoid arthritis. Stat Med 2007, 26(6):1237-1254

73. Koopman L, van der Heijden GJ, Glasziou PP, Grobbee DE, Rovers MM: A systematic review of analytical methods used to study subgroups in (individual patient data) meta-analyses. J Clin Epidemiol 2007, 60(10):1002-1009.

74. Riley RD, Lambert PC, Staessen JA, et al: Meta-analysis of continuous outcomes combining individual patient data and aggregate data. Stat Med 2008, 27(11):1870-1893.

75. Thompson SG, Higgins JP: Treating individuals 4: can meta-analysis help target interventions at individuals most likely to benefit? Lancet 2005, 365(9456):341-346.

76. Trikalinos TA, loannidis JP: Predictive modeling and heterogeneity of baseline risk in meta-analysis of individual patient data. J Clin Epidemiol 2001, 54(3):245-252.

77. Knapp G, Hartung J: Improved tests for a random effects meta-regression with a single covariate. Stat Med 2003, 22(17):2693-2710.

78. van Houwelingen $\mathrm{HC}$, Arends LR, Stijnen T: Advanced methods in meta-analysis: multivariate approach and meta-regression. Stat Med 2002, 21(4):589-624

79. Sharp SJ, Thompson SG: Analysing the relationship between treatment effect and underlying risk in meta-analysis: comparison and development of approaches. Stat Med 2000, 19(23):3251-3274.
80. Ghidey W, Lesaffre E, Stijnen T: Semi-parametric modelling of the distribution of the baseline risk in meta-analysis. Stat Med 2007, 26(30):5434-5444.

81. Cook RJ, Walter SD: A logistic model for trend in $2 \times 2 \times$ kappa tables with applications to meta-analyses. Biometrics 1997, 53(1):352-357

82. Chang BH, Waternaux C, Lipsitz S: Meta-analysis of binary data: which within study variance estimate to use? Stat Med 2001, 20(13):1947-1956.

83. Davey Smith G, Egger M, Phillips AN: Meta-analysis. Beyond the grand mean? BMJ 1997, 315(7122):1610-1614.

84. Sidik K, Jonkman JN: A note on variance estimation in random effects meta-regression. J Pharm Stat 2005, 15:823-838.

85. Sutton A: Recent development in meta-analysis. Stat Med 2008, 27:625-650.

86. Bagnardi V, Quatto P, Corrao G: Flexible meta-regression functions for modelling aggregate dose-response data, with an application to alcohol and mortality. Am J Epidemiol 2004, 159(11):1077-1086.

87. Higgins JP, Thompson SG, Deeks JJ, Altman DG: Measuring inconsistency in meta-analyses. BMJ 2007, 327:557-560.

88. Ioannidis JP: Interpretation of test of heterogeneity and bias in meta-analysis. J Eval Clin Pract 2008, 14:951-957.

89. Glasziou PP, Sanders SL: Investigating causes of heterogeneity in systematic reviews. Stat Med 2002, 21:1503-11.

90. Hatala R, Wyer P, Guyatt $G$, for the Evidence-Based Medicine Teaching Tips Working Group: Tips for learners of evidence-based medicine: 4. Assessing heterogeneity of primary studies in systematic reviews and whether to combine their results. CMAJ 2005, 172(5):661-665.

91. Bailey KR: Inter-study differences: How should they influence the interpretation and analysis of results? Stat Med. 1987, 6:351-358.

92. Khalid S, Khan RK, Kleijnen J, Antes G: Systematic Reviews to Support Evidence-based Medicine: How to Apply Findings of Health-Care Research. London: Royal Society of Medicine Press Ltd.; 2003.

93. Sutton AJ, Jones DR, Sheldon TA, Song F: Methods for Meta-analysis in Medical Research. Chichester: John Wiley \& Sons, Ltd.; 2000.

94. Littell JC, Corcoran J, Pillai VK: Systematic Reviews and Meta-Analysis. Oxford: Oxford University Press; 2008.

95. Whitehead A: Meta-Analysis of Controlled Clinical Trials. Chichester: John Wiley \& Sons, Ltd.; 2002.

96. Furlan AD, Pennick V, Bombarider C, van Tulder M, from the Editorial Board of the Cochrane Back Review Group: 2009 Updated method guidelines for systematic reviews in the Cochrane back review group. Spine 2009, 34(18):1929-1941.

97. National Health and Medical Research Council: How to Review the Evidence Systematic Identification and Review of the Scientific Literature. Australia: Commonwealth of Australia; 2000.

98. Centre for Reviews and Dissemination: CRD's Guidance for Undertaking Reviews in Health Care. York: CRD; 2009.

99. Oxman AD, Guyatt $\mathrm{GH}$ : A consumer's guide to subgroup analyses. Ann Intern Med 1992, 116(1):78-84.

100. In Cochrane Handbook for Systematic Reviews of Interventions. Version 5.0.0 [updated September 2008]. Edited by Higgins JPT, Green S.: The Cochrane Collaboration; 2008. Available from: www.cochrane-handbook.org.

101. Imperiale TF: Meta-analysis: when and how. Hepatology 1999, 29(6 Suppl):26S-31S.

102. Shekelle PG, Morton SC: Principles of metaanalysis. J Rheumato/ 2000 27(1):251-252. discussion 252-53.

103. Nagin DS, Odgers CL: Group-based trajectory modeling in clinical research. Annu Rev Clin Pscyhol. 2010, 6:109-138.

104. Virgile G, Conto AA, Moja L, Gensini GL, Gusinu R: Heterogeneity and meta-analyses: do study results truly differ? Intern Emerg Med 2009, 4:423-427.

105. Skipka G, Bender R: Intervention effects in the case of heterogeneity between three subgroups: Assessment within the framework of systematic reviews. Methods Inf Med. 2010, 49:613-617.

106. Groenwold RHH, Rovers MM, Lubsen J, van der Heijden JMG: Subgroup effects despite homogenous heterogeneity test results. BMC Medical Research Methodology. 2010, 10:43.

107. Lockwood CM, DeFrancesco CA, Elliot DL, Beresford SAA, Toobert DJ: Mediation analyses: Applications in nutrition research and reading the literature. J Am Diet Assoc 2010, 110:753-763. 
108. Baker W, White M, Cappelleri JC, Kluger J, Colman Cl: Understanding heterogeneity in meta-analysis: the role of meta-regression. Int $J$ Clin Pract 2009, 63(10):1426-1434.

109. Jones AP, Riley RD, Williamson PR, Whitehead A: Meta-analysis of individual patient data versus aggregate data from longitudinal clinical trials. Clin Trials 2009, 6:16-27.

110. Hemming K, Hutton JL, Maguire MJ, Marson AG: Meta-regression with partial information on summary trial or patient characteristics. Stat Med 2008, 29:1312-1324

111. Salanti G, Marinho V, Higgins JPT: A case study of multiple-treatments meta-analysis demonstrates covariates should be considered. I Clin Epidemiol 2009, 62:857-864.

112. Glasziou P, Chalmers I, Altman DG, Bastian H, Boutron I, Brice A, et al: Taking healthcare interventions from trial to practice. BMJ 2010, 341:c3852.

113. Shadish WR: Meta-analysis and the exploration of causal mediating processes: A primer of examples, methods, and issues. Psychol Methods 1996, 1:47-65.

114. Borenstein MA, Hedges LV, Higgins JPT, Rothstein HR: Introduction to Meta-Analysis. New Jersey: John Wiley and Sons; 2009.

115. West SL, Gartlehner G, Mansfield AJ, et al: Comparative effectiveness review methods: clinical heterogeneity, In: Agency for Healthcare Research and Quality. Comparative Effectiveness Review Methods: Clinical Heterogeneity. Rockville, MD:; Posted 09/28/2010. Available at http://effectivehealthcare. ahrq.gov/.

116. Mclntosh MW: The population risk as an explanatory variable in research synthesis of clinical trials. Stats Med 1996, 15:1713-1728.

117. Boutitie F, Gueyffier F, Pocock SJ, Biossel JP: Assessing treatment-time interaction in clinical trials with time to event data: A meta-analysis of hypertension trials. Stat Med 1998, 17:2883-2903.

118. Booth A, Clarke M, Ghersi D, MOher D, Petticrew M, Stewart L: An international registry of systematic-review protocols. Lancet 2011, 377(9760):108-109

119. Concato J, Peduzzi P, Holfold TR, et al: Importance of events per independent variable in proportional hazards analysis. I. Background, goals, and general strategy. J Clin Epidemiol 1995, 48:1495-1501.

120. Peduzzi P, Concato J, Feinstein AR, et al: Importance of events per independent variable in proportional hazards regression analysis. II. Accuracy and precision of regression estimates. J Clin Epidemiol 1995, 48:1503-1510

121. Peduzzi $P$, Concato J, Kemper $E$, et al: A simulation study of the number of events per variable in logistic regression analysis. J Clin Epidemiol 1996, 49:1373-1379.

122. Harrell FE: Regression Modeling Strategies: With Applications to Linear Models, Logistic Regression, and Survival Analysis. New York: Springer; 2001.

123. Spiegelhalter DJ, Myles JP, Jones DR, Abrams KR: Bayesian methods in health technology assessment: A review. Health Technol Assess 2000, 4:1-130.

124. Smith CT, Williamson PR, Marson AG: An overview of methods and empirical comparison of aggregate data and individual patient data results for investigating heterogeneity in meta-analysis to time-to-event data. J Eval Clin Pract 2002, 55:86-94.

125. Lambert PC, Sutton AJ, Jones ADR: A comparison of patient-level covariates in meta-regression with individual patient data meta-analysis. J Clin Epidemiol 2002, 55:86-94.

doi:10.1186/1471-2288-12-111

Cite this article as: Gagnier et al: Investigating clinical heterogeneity in systematic reviews: a methodologic review of guidance in the literature. BMC Medical Research Methodology 2012 12:111.

\section{Submit your next manuscript to BioMed Central and take full advantage of:}

- Convenient online submission

- Thorough peer review

- No space constraints or color figure charges

- Immediate publication on acceptance

- Inclusion in PubMed, CAS, Scopus and Google Scholar

- Research which is freely available for redistribution

Submit your manuscript at www.biomedcentral.com/submit
Biomed Central 